ISSN 1112-9867

http://www.jfas.info

\title{
INVOLVEMENT OF SNP MARKER LOCATED ON THE CALCIUM BINDING PROTEIN GENE IN ADAPTIVE TRAITS AND ORGANOLEPTIC PERFORMANCES OF THE OLIVE TREE
}

\author{
R. Ben Ayed*, K. Ennouri, A. Rebai \\ Laboratoire de Criblage Moléculaire et Cellulaire, Centre de Biotechnologie de Sfax, Tunisie.
}

Received: 24 March 2017 / Accepted: 29 December 2017 / Published online: 01 January 2018

\begin{abstract}
Olive trees importance is mainly due to the economic and health benefits, especially in the Mediterranean basin. Unfortunately, to enhance productivity and quality of olive oil, the study of both molecular and phenotypic characterizations of olive cultivars is crucial. We consider the analysis of 14 Tunisian olive cultivars of known origin sampled from different areas of the country based on SNP marker (CALC) located in calcium binding protein gene. Accordingly, we assessed the potential genotype-phenotypes links between the SNP, on the one hand, and the quantitative and qualitative parameters, on the other. The statistical analysis based on bivariate analysis for the CALC marker suggests that Chemlali varieties with CG genotypes have better capacity to produce fruit, fruit oil, oil content in carotene, cholesterol and linoleic acid than homozygote GG genotypes varieties. These results are confirmed by a multivariate logistic regression.
\end{abstract}

Keywords: Olea europaea L; CALC; calcium binding protein; bivariate analysis; multivariate logistic regression.

Author Correspondence, e-mail: raydabenayed@yahoo.fr

doi: http://dx.doi.org/10.4314/jfas.v10i1.24 


\section{INTRODUCTION}

Les plantes cultivées peuvent être confrontées à des contraintes environnementales qui risquent d'être néfastes pour leur développement et pouvant entrainer des conséquences allant de la baisse de rendement jusqu'à la perte totale des récoltes. L'olivier (Olea europaea L.) est un arbre robuste, typiquement méditerranéen, que l'on cultive depuis l'Antiquité pour ses fruits et l'huile d'olive qu'il produit. Cependant, bien que la plupart des variétés d'olivier résistent bien à la sécheresse estivale, au froid et à la salinité, ce n'est pas le cas pour certaines variétés [1].

La réponse adaptative des plantes repose sur des bases physiologiques et moléculaires variées et commence généralement par des mécanismes cellulaires de signalisation du stress [2]. Au delà de son rôle, dans la nutrition des plantes en tant que macroélément et son implication dans la structure de la paroi de la cellule végétale, le calcium est connu pour moduler de nombreux aspects du développement, de la croissance et des réponses d'adaptation aux contraintes de l'environnement [3]. En effet, le calcium est un second messager essentiel qui centralise les réponses à divers signaux développementaux et environnementaux. Les signatures calciques, spécifiques de chaque stimulus, sont perçues et décodées par divers senseurs de calcium qui induisent alors les réponses appropriées [4]. Pour maintenir le gradient de concentration du calcium entre le cytoplasme et le milieu extérieur, il faut un efflux du calcium hors de la cellule contre son gradient électrochimique. Le transport de calcium est effectué par les protéines de la famille des calmodulines [5]. La calmoduline (ou «calcium binding protein ») est le médiateur de calcium le plus important et le mieux conservé chez les eucaryotes. La fixation du calcium sur les senseurs induit un changement conformationnel qui modifie alors leur interaction avec des partenaires ou leur activité enzymatique [3]. Les senseurs de calcium ainsi activés régulent leurs protéines cibles qui peuvent être impliquées dans la transduction du signal, comme des protéines kinases ou des facteurs de transcription [5]. Les protéines cibles peuvent être directement impliquées dans la protection cellulaire face aux dommages induits par les stress, comme des transporteurs d'ions ou des enzymes de détoxication. Le calcium joue un rôle important dans la signalisation de la perturbation osmotique induite par le froid, la sécheresse et la salinité [3]. La multiplicité des 
senseurs de calcium végétaux associées aux diverses cibles cellulaires constituent un réseau de signalisation finement régulé qui induit des réponses de stress spécifiques et améliore la survie des plantes dans un environnement défavorable. Pour les plantes, les calmodulines jouent un rôle dans le système de défense contre les conditions stressantes [6;7].

L'objectif de ce travail est d'étudier un marqueur SNP localisé au niveau du gène calcium binding protein afin de classifier 14 variétés d'olivier tunisiennes d'une part et de valider la relation génotype-phénotype entre ce marqueur et quelques paramètres quantitatifs et qualitatifs d'autre part, pouvant aboutir à l'identification d'éventuelles associations entre variabilité moléculaire et paramètres de qualité des huiles.

\section{RESULTS AND DISCUSSION}

\subsection{Optimisation de la réaction PCR}

L'efficacité d'une réaction PCR dépend fortement du choix des amorces, nous avons conçu un couple d'amorces. Nous avons choisi un ADNc comprenant le locus d'intérêt $(\mathrm{C} / \mathrm{G})$ et disponible dans le site NCBI (http://www.ncbi.nlm.gov/). Les couples d'amorces permettant d'amplifier les loci d'intérêt ont été conçus à l'aide du logiciel Primer3. Les paramètres suivants ont été sélectionnés pour toutes les amorces: température de fusion théorique (Tm ) proche de $66^{\circ} \mathrm{C}$, composition équilibrée entre les bases $\mathrm{A}$ et $\mathrm{T}$ d'une part et $\mathrm{C}$ et $\mathrm{G}$ d'autre part, longueur des amorces comprise entre 18 et 30 nucléotides et une à trois bases $\mathrm{G}$ et $\mathrm{C}$ parmi les 7 dernières bases à partir de l'extrémité 3' de l'amorce. Par ailleurs, seuls les couples d'amorces générant un amplicon de taille $476 \mathrm{pb}$ ont été conservés pour que même les fragments d'ADN très dégradés puissent être amplifiés. La spécificité des amorces choisies a été testée en comparant la séquence de chaque amorce à la base de données Genbank grâce au logiciel BLASTn (http://blast.ncbi.nlm.nih.gov/). Finalement, nous avons vérifié que les amorces choisies ne formaient pas de structures secondaires telles que des dimères d'amorces ou des épingles à cheveux. Le couple d'amorce conservé pour ce marqueur est présenté dans le tableau 1. 
Tableau 1. Noms du gène, du marqueur SNP, des amorces et leurs séquences.

\begin{tabular}{llll}
\hline Nom complet du gène & $\begin{array}{c}\text { Nom des } \\
\text { amorces }\end{array}$ & Nom du SNP & Séquences des amorces \\
\hline Calcium binding protein & $\begin{array}{l}\text { CALC-F } \\
\text { CALC-R }\end{array}$ & CALC & $\begin{array}{l}5^{\prime} \text { ' cacggacaggaattccaagccttca 3' } \\
5, \text { tgccgcttttgttcgtcatcattttct 3' }\end{array}$ \\
\hline
\end{tabular}

La détection du marqueur SNP CALC a été réalisée par digestion de ses produits PCR en utilisant une enzyme de restriction spécifique BstzI. Les fragments attendus de ce SNP, en présence du site de restriction, sont rapportées dans la figure 1 du gel d'électrophorèse.

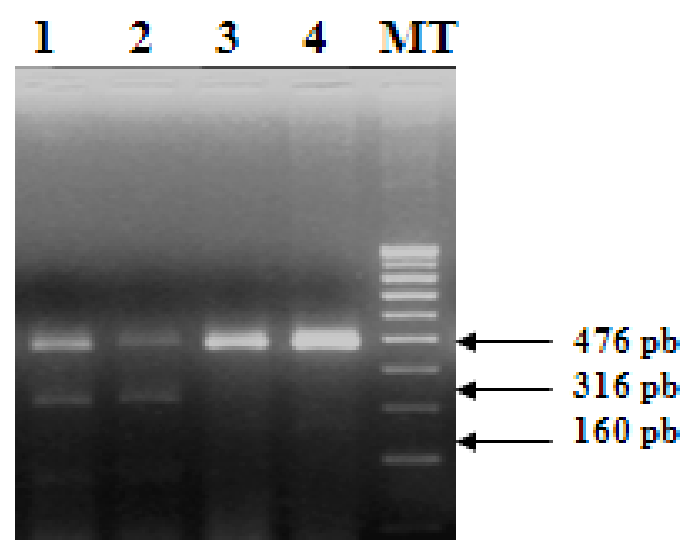

Fig.1. Produits de digestion du SNP de type CALC testés sur gel d'agarose 3\%.

1: Oueslati; 2: Chemlali Tataouine; 3: Zarrazi Zarzis; 4 : Chetoui Nabeul, MT : Marqueur de taille (100 bp DNA Step Ladder, Promega)

\section{2 Caractéristiques des SNPs étudiés}

Le marqueur CALC est de type transversion $(C \rightarrow G)$. Le calcul des fréquences alléliques ainsi que des fréquences génotypiques du marqueur CALC nous ont montré que l'allèle $\mathrm{G}$ de CALC paraît prédominant dans notre échantillon de variétés d'olivier, ce qui suppose qu'il pourrait être un allèle ancestral (Figure2).

Le calcul des fréquences génotypiques montre la présence de deux génotypes pour les deux SNP CALC, ce résultat est confirmé par Reale et al. (2006) [8]. 


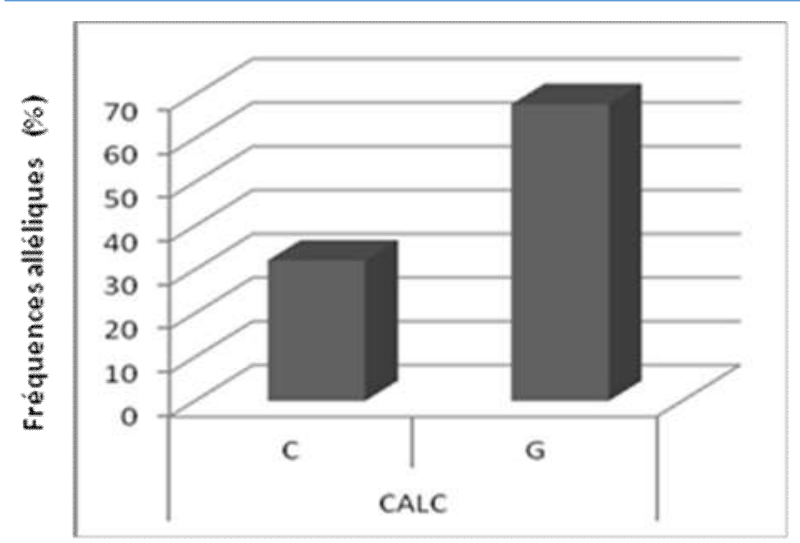

A

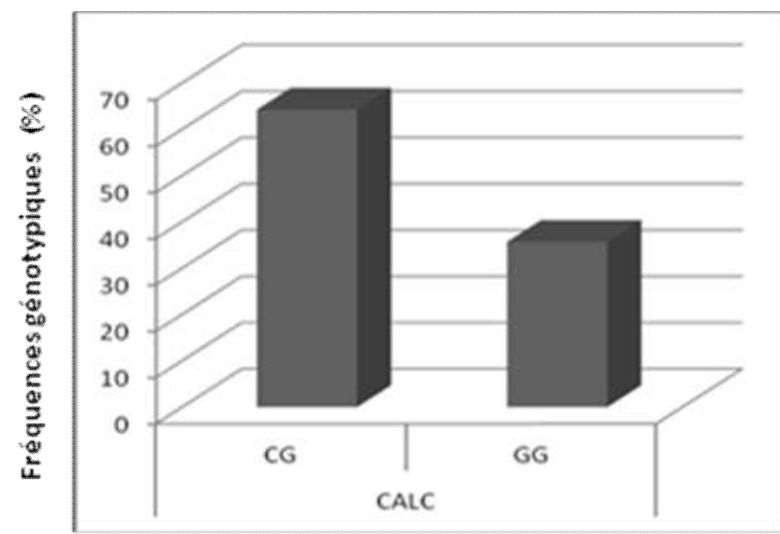

B

Fig.2. Les fréquences alléliques (A) et génotypiques (B) du marqueur CALC

D'après les résultats (Tableau 2), l'hétérozygotie observée est de 0,727 (CALC). On remarque que l'hétérozygotie observée est supérieure à celle attendue.

Le pouvoir de discrimination (PD) varie de 0,396 pour le marqueur CALC. Des valeurs similaires de pouvoir de discrimination ont été trouvées par Reale et al. (2006) [8].

Pour les fréquences alléliques de chaque marqueur SNP étudié, nous avons remarqué qu'il y a une dominance d'un allèle par rapport à l'autre.

Tableau 2. Caractéristiques du marqueur CALC chez 14 variétés d'olivier.

\begin{tabular}{lllllllll}
\hline Locus & $\begin{array}{c}\text { Type de } \\
\text { polymorphisme }\end{array}$ & $\mathbf{T m}^{\mathbf{a}}$ & $\begin{array}{c}\text { Longueurs } \\
\text { des bandes } \\
\text { en pb }\end{array}$ & Ho & He & PD & r \\
\hline CALC & G/C & 60 & 476 & 0,727 & 0,462 & 0,396 & $-0,180$ \\
\hline
\end{tabular}

Pour le locus CALC, la taille des allèles en paires de base, l'hétérozygotie observée (Ho), l'hétérozygotie attendue (He), le pouvoir de discrimination (PD), et la probabilité d'allèles nuls (r) sont signalés. ${ }^{\text {a}}$ : température d'hybridation pour chaque PCR.

\section{3 Analyse du dendrogramme}

Deux groupes de variétés peuvent être définis en coupant le dendrogramme à une distance de 0,5 (Figure 3). Le groupe 1 comprend 5 cultivars, 'Chemlali' (Chem_SB, Chem_Sous, Chem_Dok, Chem_Nab et Chem_Mon), 'Chem_Tat', 'Zalmati', 'Chemch' et 'Oueslati'. Le même groupe a été trouvé au niveau du dendrogramme basé sur les marqueurs SSR. Le deuxième groupe contient 'Zarrazi', les trois cultivars 'Chetoui' (Chetoui_Silia, Chet_Thibar 
et Chet_Nab) et 'Rkhaymi'.

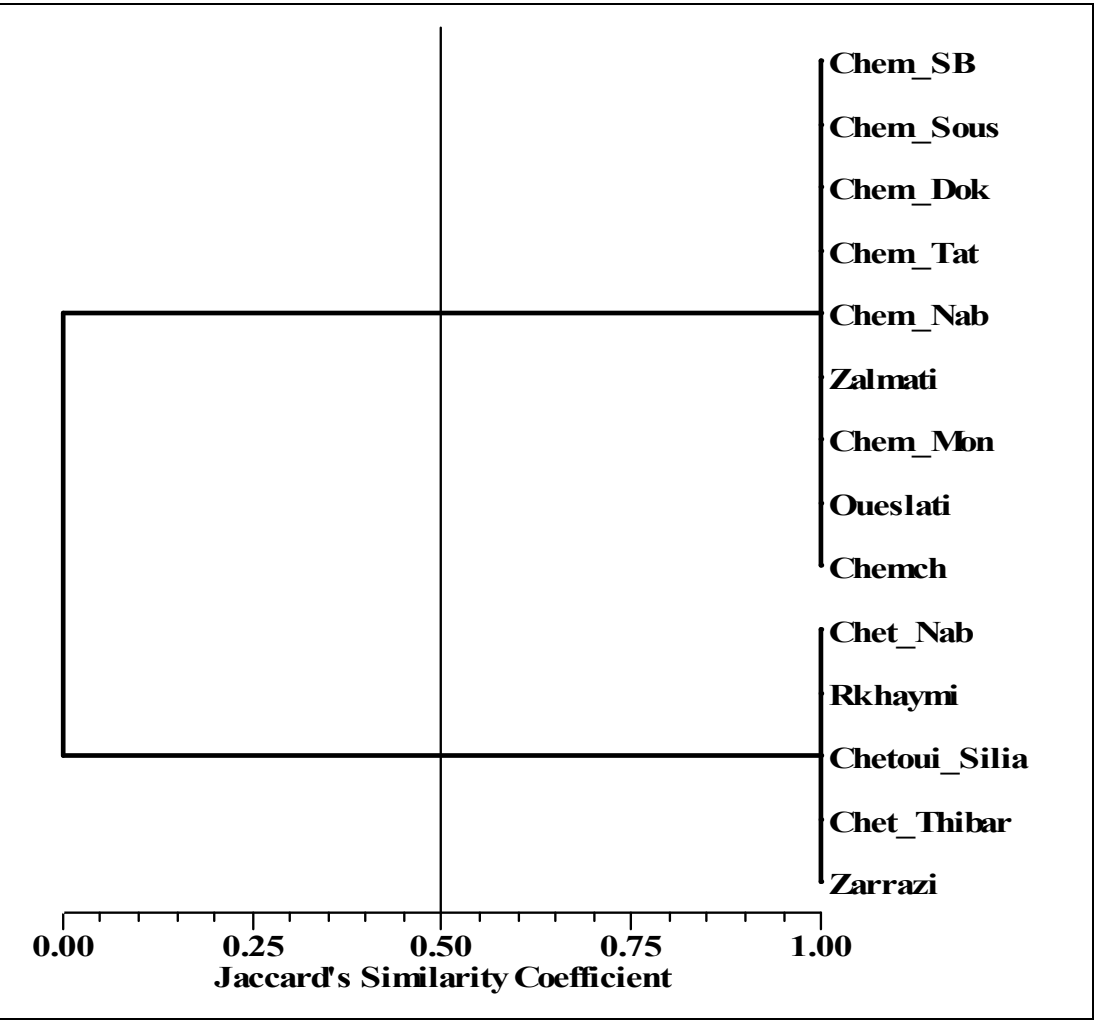

Fig.3. Dendrogramme des génotypes des 14 variétés étudiées par le marqueur SNP CALC en utilisant le programme NTSYS-pc (version 2.1) et analyse par la méthode UPGMA.

\section{4 Association génotype-phénotype du SNP étudié}

\subsubsection{Relation entre le marqueur SNP CALC et les paramètres qualitatifs}

Dans cette partie, nous avons étudié la relation potentielle entre un marqueur SNP CALC et quelques paramètres qualitatifs que nous avons estimés importants dans le cas de l'huile d'olive. Ces caractères concernent essentiellement le niveau de maturation des fruits ainsi que la forme du fruit, la pollinisation (auto-compatible ou auto-incompatible), la teneur en huile par fruit, la productivité et finalement la tolérance de l'arbre implanté dans des conditions de stress mesurée notamment par des classes reflétant la tolérance au froid, à la salinité et la sécheresse. Des associations hautement significatives ont été observées pour le CALC avec le niveau de maturation de fruit et la tolérance, respectivement $\left(\chi^{2}=10,516 ; p=0,005\right)$ et $\left(\chi^{2}=10,128 ; p=0,006\right)$. D'autres associations positives ont été observées pour le CALC avec la teneur en huile/fruit (Tableau 3).

Marqueur CALC et maturation de fruit: dans notre étude, il ressort que le niveau de 
maturation est étroitement dépendant de la variation génotypique du marqueur CALC. En effet, le niveau tardif de la maturation de l'olive est observé essentiellement pour le génotype CG $(57,14 \%)$ du marqueur CALC pour toutes les variétés tunisiennes, alors que pour le génotype GG, seulement $28,57 \%$ des variétés étudiées ont une maturation moyenne, mais aucune variété portant le génotype (GG) n'a donné une maturation tardive. De ce fait, on peut suggérer pour ce marqueur que l'allèle C est étroitement associé avec le retard de la maturation de l'olive.

Marqueur CALC et teneur en huile du fruit: la teneur en huile par fruit semble être associée à la variation génotypique du marqueur CALC. En effet, 42,85\% des variétés tunisiennes étudiées qui portent le génotype CG ont une teneur en huile par fruit relativement élevée, tandis qu'aucune variété portant le génotype GG ne montre une teneur importante d'huile par fruit. Ainsi, pour ce marqueur type SNP (CALC), on suggère que l'allèle C est étroitement associé à l'augmentation de la teneur d'huile/olive.

Marqueur CALC et productivité: la productivité de l'olivier est associée à la variation génotypique de CALC. En effet, 42,85\% des variétés tunisiennes étudiées qui portent le génotype $\mathrm{CG}$ ont une productivité élevée et alternante par rapport au reste des variétés rencontrées dans ce travail. Les variétés dotées d'une productivité importante et d'un génotype CG du marqueur CALC regroupe essentiellement les variétés 'Chemlali' et 'Chetoui'. Toutefois, pour le génotype GG, on note que $14,28 \%$ des variétés étudiées ont une productivité faible ou moyenne et alternante, sans qu'aucune variété ne soit en mesure de donner une productivité élevée et alternante pour ce génotype (GG). Par conséquent, l'allèle $\mathrm{C}$ de ce marqueur serait étroitement associé avec le niveau élevé de productivité.

Marqueur CALC et tolérance générale de l'arbre: suite à l'analyse de la variation génotypique du marqueur CALC, nous avons remarqué la présence d'un lien entre la capacité de l'olivier à tolérer des conditions défavorables à sa croissance (salinité, sécheresse ou froid) et le variant CG du marqueur CALC. En effet, 61,53\% des variétés tunisiennes étudiées et qui portent le génotype CG tolèrent la sécheresse et la salinité, alors que pour ce génotype aucune variété qui supporte le froid ou le froid et la salinité n'a été observée. Environ 23,07\% des variétés étudiées et qui portent le génotype (GG) supportent le froid et la salinité mais aucune variété portant ce génotype (GG) et tolérante à la sécheresse et la salinité n'a été observée. Par 
conséquent, pour ce marqueur, probablement l'allèle C pourrait être corrélé à la capacité de l'olivier à tolérer la sécheresse et la salinité.

Les associations génotypiques positives établies dans notre étude entre le marqueur CALC et la capacité de l'olivier à tolérer des conditions stressantes de sécheresse, salinité et froid, sont biologiquement raisonnables et concordent avec les données de la littérature. En effet, la relation entre le calcium et le comportement de la plante vis à vis de quelques conditions stressantes est bien établi dans la littérature [9]. Rappelons que les calmodulines sont impliquées dans le mécanisme de défense de la plante contre les conditions climatiques stressantes. La calmoduline est une protéine capable de percevoir les variations du taux de calcium par des motifs de type EF-hand, qui ont une affinité élevée pour cet ion. Le calcium joue un rôle important dans la signalisation osmotique induite par le froid, la sécheresse et la salinité. La multiplicité des senseurs de calcium végétaux associés aux diverses cibles cellulaires constituent un réseau de signalisation finement régulé qui induit des réponses au stress d'une manière très spécifique dans le but d'augmenter la survie des plantes et d'améliorer leur capacité d'adaptation dans un environnement défavorable [10]. En effet, la sur-expression d'un antiport $\mathrm{H}^{+} / \mathrm{Ca}^{2+}$ au niveau du tonoplaste du tabac (CAX1 d'Arabidopsis) entraîne l'incapacité de la plante à réagir de façon normale au froid du fait de la modification de l'homéostasie du calcium cytoplasmique [6]. En outre, beaucoup de travaux sur les calmodulines végétales ont contribué à révéler des liens étroits entre l'expression génique et la signalisation calcique par les rôles directs ou indirects de leurs cibles nucléaires sur la régulation de la transcription et d'événements post-transcriptionnels. 
Tableau 3. Distribution génotypique du marqueur CALC et leurs associations aux paramètres qualitatifs.

\begin{tabular}{|c|c|c|c|c|c|}
\hline \multirow{2}{*}{\multicolumn{2}{|c|}{ Paramètres qualitatifs }} & \multicolumn{4}{|c|}{ CALC } \\
\hline & & \multirow{2}{*}{$\frac{\mathbf{C G}(\%)}{0}$} & \multirow{2}{*}{$\begin{array}{c}\text { GG (\%) } \\
7,14\end{array}$} & \multirow[t]{2}{*}{$\chi^{2}$} & \multirow{2}{*}{$\frac{p}{0,005}$} \\
\hline Maturation & Précoce & & & & \\
\hline \multirow[t]{3}{*}{ du fruit } & Moyenne & 7,14 & 28,57 & 10,516 & \\
\hline & Tardive & 57,14 & 0 & & \\
\hline & Faible & 0 & 7,14 & & \\
\hline Teneur & Moyenne & 0 & 21,42 & 10,733 & 0,013 \\
\hline \multirow[t]{2}{*}{ huile/fruit } & élevée & 42,85 & 0 & & \\
\hline & Très élevée & 21,42 & 7,14 & & \\
\hline \multirow[t]{5}{*}{ Productivité } & Faible & 0 & 7,14 & & \\
\hline & Faible et alternante & 0 & 7,14 & & 0,085 \\
\hline & Moyenne et alternante & 7,14 & 14,28 & 8,193 & \\
\hline & Elevée et alternante & 42,85 & 0 & & \\
\hline & Très élevée & 14,28 & 7,14 & & \\
\hline \multirow[t]{4}{*}{ Tolérance } & Froid & 0 & 7,69 & & \\
\hline & Froid et salinité & 0 & 23,07 & 10,128 & 0,006 \\
\hline & Sécheresse & 0 & 7,69 & & \\
\hline & Sécheresse et salinité & 61,53 & 0 & & \\
\hline
\end{tabular}

$P$ : P-valeur du test Chi deux

\subsubsection{Relation entre les marqueurs génétiques et les paramètres quantitatifs}

Dans cette étude, huit paramètres quantitatifs ont été évalués pour les 16 variétés tunisiennes qui ont fait l'objet de notre analyse de l'interaction génotypes-phénotypes. Des tests statistiques de comparaisons de moyennes (test de Student) ont été menés pour étudier les associations potentielles du marqueur génétique du gène Calcium binding protein et l'ensemble des paramètres phénotypiques à caractères quantitatifs. Parmi ces derniers, nous avons choisi de focaliser sur la teneur de l'huile d'olive en acides gras polyinsaturés (C18:2 et $\mathrm{C} 18$ :3), la teneur en carotène, la teneur en chlorophylle, la teneur en cholestérol, betasitostérol et la teneur en composés poly-phénoliques.

Une différence significative de la moyenne de la teneur en carotène entre les variétés au génotype CG (moyenne: 5,736 $\pm 1,35$ ) et au génotype GG (moyenne: 3,84 $\pm 0,08$ ) du marqueur CALC $(p=0,008)$ a été observée. De même, nous avons noté une relation positive entre le taux de cholestérol et la variation génotypique pour ce marqueur $(p=0,025)$, dans ce cas, ce sont les variétés aux génotypes CG qui ont un taux plus élevé de cholestérol. Une troisième 
association faiblement significative du variant CG du marqueur CALC avec le taux d'acides gras insaturés $(\mathrm{C} 18: 3)$ a été prouvée $(p=0,037)$. Ces trois associations positives pour le CALC et la teneur en carotène, en cholestérol et en acide linoléique, laisse suggérer que les variétés hétérozygotes aux génotypes CG sont meilleures que celles aux génotypes homozygotes GG au niveau de leur capacité à produire ces substances (pigment de carotène, béta-sitostérol et l'acide gras insaturé (Tableau 4).

Tableau 4. Association entre les génotypes CALC et les paramètres quantitatifs de l'huile d'olive.

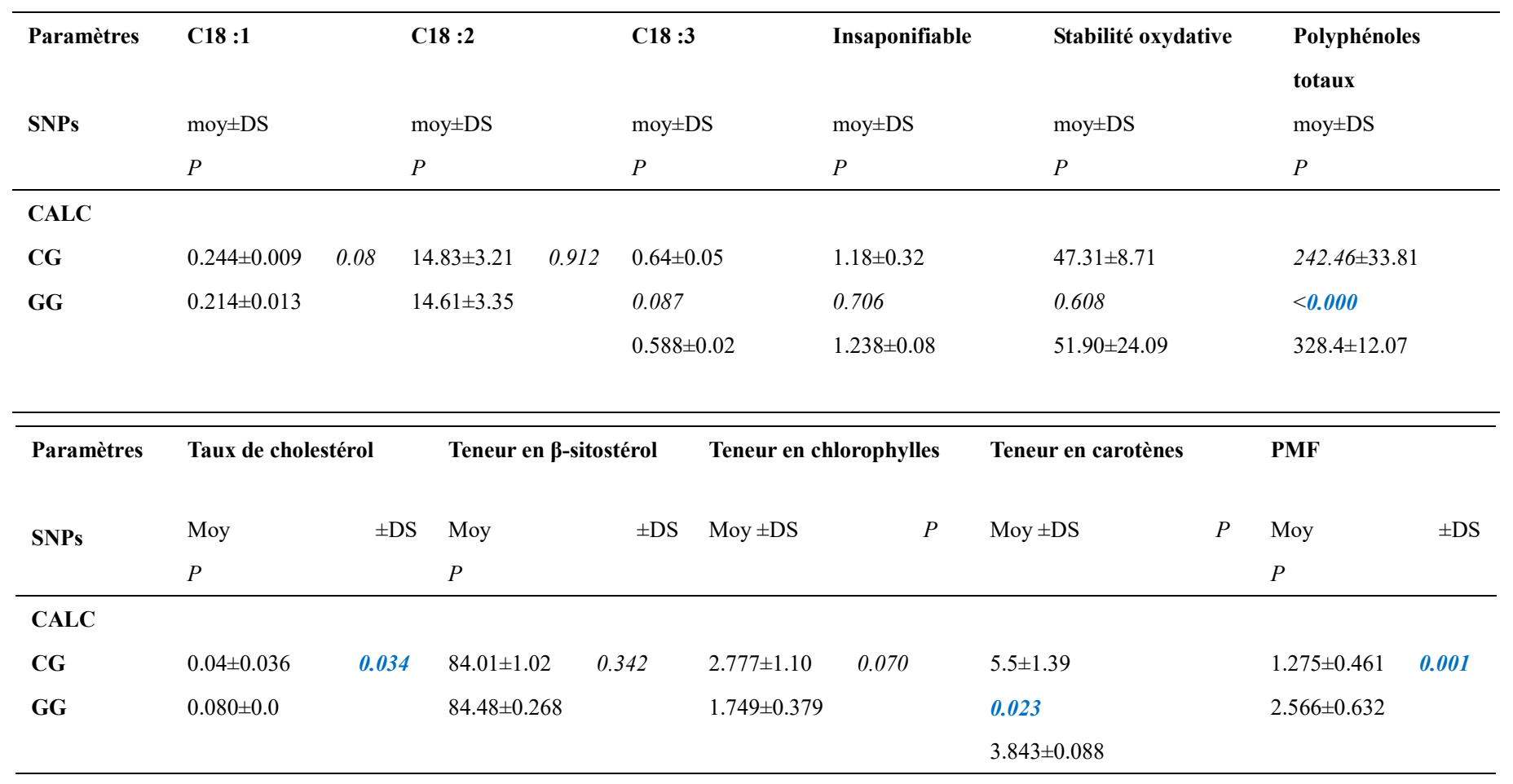

P: P-valeur du test Student; Moyenne: moyenne de score d'expression; SD: Déviation Standard

\subsubsection{Analyse multivariée par la régression logistique binaire}

L'association CALC avec la teneur en carotènes est confirmée par l'analyse de régression logistique en utilisant les génotypes des marqueurs comme variables dépendantes et les paramètres de l'huile comme variables explicatives (Tableau 5). On remarque aussi que parmi les variables qualitatives, seule la tolérance de l'arbre possède une association significative avec le marqueur CALC, ce qui conforte la conclusion de l'analyse bivariée. 
Tableau 5. Analyse multivariée par régression logistique binaire.

\begin{tabular}{lc}
\hline Modèle & CALC** \\
& \\
\hline PMF & $\mathbf{0 . 0 0 2}$ \\
Acidité & 0.584 \\
Teneur en polyphénols totaux $(\mathbf{m g} / \mathbf{k g})$ & $\mathbf{0 . 0 0 2}$ \\
Taux d'insaponifiable\% & 0.705 \\
Stabilité oxydative $\left(\mathbf{h}\right.$ à $\left.\mathbf{1 0 0}{ }^{\circ} \mathbf{C}\right)$ & $\mathbf{0 . 0 0 4}$ \\
Teneur en chlorophylles $(\mathbf{p p m})$ & $\mathbf{0 . 0 9 0}$ \\
C18 :2 & 0.903 \\
C18 :3 & $\mathbf{0 . 0 7 4}$ \\
Cholestérol & $\mathbf{0 . 0 1 2}$ \\
Teneur en carotènes (ppm) & $\mathbf{0 . 0 1 7}$ \\
$\mathbf{\beta}$-sitostérol & 0.103 \\
Maturation du fruit & $\mathbf{0 . 0 0 2}$ \\
Utilisation & $\mathbf{0 . 0 0 1}$ \\
Forme du fruit & 0.101 \\
Pollinisation & 0.101 \\
Tolérance de l'arbre & $\mathbf{0 . 0 0 2}$ \\
Teneur en huile par fruit & 0.071 \\
Productivité de l'arbre & $\mathbf{0 . 0 4 4}$ \\
**: on donne les p-valeurs dans l'analyse de régression logistique binaire
\end{tabular}

Cette analyse multivariée confirme les relations déjà identifiées dans les analyses bivariées comme par exemple celles de CALC avec la tolérance de l'arbre. Ces résultats sont biologiquement appropriés vu que ce marqueur est localisé dans le gène Calcium Binding Protein "Calc» qui joue un rôle dans le système de défense contre les conditions stressantes chez les plantes, ce qui pourrait expliquer l'association entre ce marqueur et la tolérance de l'arbre.

\section{EXPERIMENTAL}

\section{1 Matériel biologique}

Quatorze génotypes tunisiens d'olivier ont été sélectionnés dans différentes régions géographiques du pays allant du nord au sud et couvrant ainsi plusieurs étages bioclimatiques (Tableau 6, Fig.3). 
Tableau 6. Liste des quatorze variétés étudiées et leurs localités.

\begin{tabular}{|c|c|c|c|}
\hline Variétés & Abréviation & $\begin{array}{c}\text { Poids moyen } \\
\text { du fruit (g) }\end{array}$ & Région \\
\hline Chemlali Sidi Bouzid & Chem_SB & 1 & Sidi Bouzid \\
\hline Chemlali Sousse & Chem_Sous & 1 & Sousse \\
\hline Chemlali Dokhane & Chem_Dok & 1 & Sfax (Dokhane) \\
\hline Chemlali Monastir & Chem_Mon & 1 & Monastir \\
\hline Chemlali Nabeul & Chem_Nab & 1 & Nabeul \\
\hline Zalmati Zarzis & Zalmati & 1,2 & Zarzis (Sud de la Tunisie) \\
\hline Chemcheli Gafsa & Chemch & 3 & Gafsa (Centre de la Tunisie) \\
\hline Oueslati Kairouan & Oueslati & 1,6 & Kairouan (Centre de la Tunisie) \\
\hline Zarrazi Zarzis & Zarrazi & 3 & Zarzis (Sud de la Tunisie) \\
\hline Chemlali Tataouine & Chem_Tat & 0,8 & Tataouine (Sud de la Tunisie) \\
\hline Chetoui Thibar & Chet_Thibar & 2,8 & Béja (Nord de la Tunisie) \\
\hline Chetoui Siliana & Chetoui_Silia & 2,8 & Siliana \\
\hline Chetoui Nabeul & Chet_Nab & 2,8 & Nabeul \\
\hline Rkhaymi & Rkhaymi & 1,6 & Nabeul \\
\hline
\end{tabular}

Dokhane: est situé à $20 \mathrm{Km}$ au Nord de Sfax

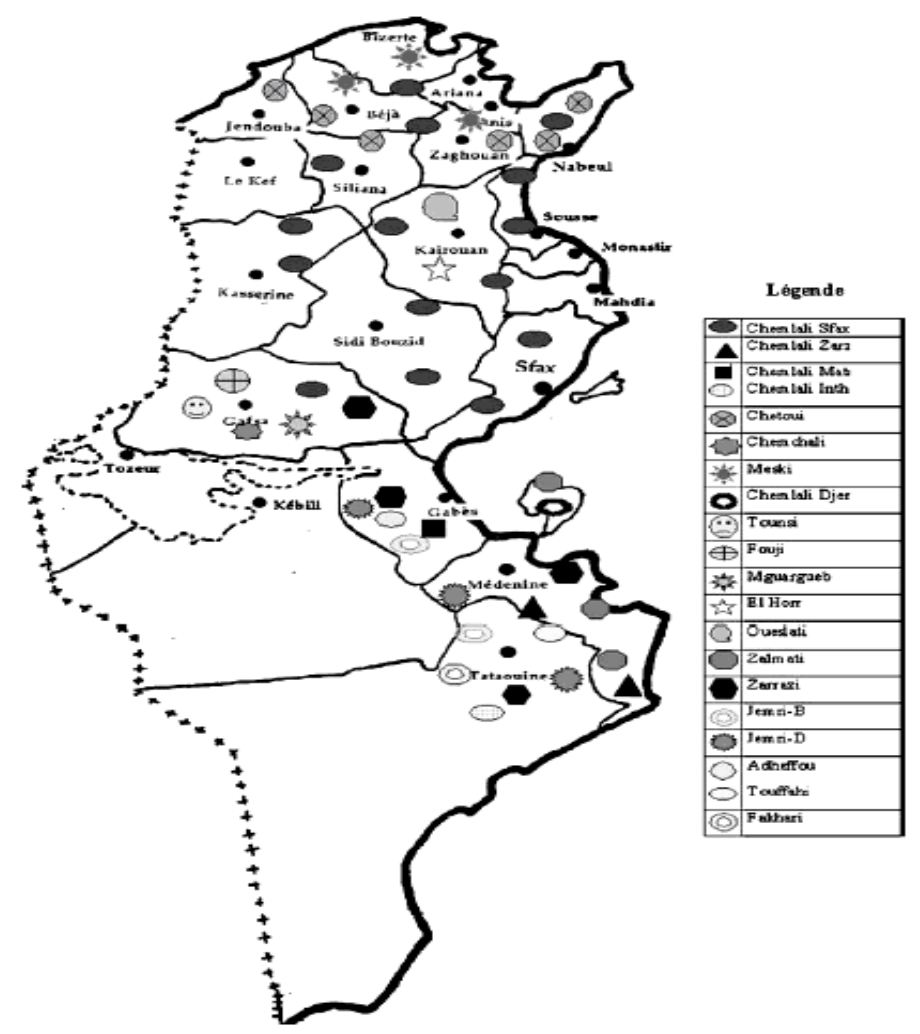

Fig.3. Localisations géographiques de chaque variété.

\section{2 Extraction de l'ADN génomique de l'olivier}

De jeunes feuilles fraîchement prélevées sont utilisées pour extraire l'ADNg en utilisant le 
protocole CTAB comme décrit par Fabbri et al. (1995) [11] et auquel nous avons apporté une légère modification [12]. L'ADN génomique (non dilué) a été stocké dans un tampon TE (1X $\mathrm{pH} 8,0)$ à $-20^{\circ} \mathrm{C}$.

\section{3 Conception des amorces PCR}

Nous avons choisi l'ADNc codant pour l'enzyme «Calcium Binding Protein » (numéro d'accession: AF078679) à partir de la base de donnée publique GeneBank du site NCBI (http://www.ncbi.nlm.nih.gov/).

Nous avons désigné des amorces pour cet ADNc à l'aide du programme Primer 3 (http://bioinfo.ut.ee/primer3-0.4.0/) afin d'amplifier une partie de l'ADNc choisi et correspondant au site du SNP. Après une étape d'optimisation, nous avons réalisé une amplification par PCR des séquences en utilisant les amorces nouvellement conçues dans cette étude.

La détection du marqueur SNP CALC a été réalisée par digestion de ces produits PCR en utilisant l'enzyme de restriction spécifique BstzI (10 U/ $\mu$ l) commercialisé par Proméga. Les sites de de restriction ont été déterminés à l'aide du programme REBASE (http://rebase.neb.com/rebase/rebase.html ).

\section{4 Amplification par PCR}

La validation du couple d'amorces PCR a consisté à l'amplification des fragments contenant le marqueur SNP d'intérêt au moyen de la réaction de PCR suivie d'une digestion moyennant une enzyme de restriction.

La réaction de PCR est réalisée dans un volume réactionnel de $30 \mu 1$ dans un thermocycleur de type GeneAmp PCR System 9700 (PERKIN-ELMER), contenant : 10 $\mu 1$ Tampon Promega (5X), $2.5 \mu 1 \mathrm{MgCl}_{2}(25 \mathrm{mM}), 2 \mu \mathrm{l} \mathrm{dNTP}(10 \mathrm{mM}), 1 \mu 1$ d'oligonucléotide sens $(10 \mu \mathrm{M}), 1 \mu 1$ d'oligonucléotide anti-sens $(10 \mu \mathrm{M}), 10 \mu \mathrm{l}$ d'ADN $(10 \mathrm{ng} / \mu \mathrm{l}), 0.25 \mu 1$ Taq polymérase $\quad(5 \mathrm{U} / \mu \mathrm{l})$ et $3.25 \mu 1 \mathrm{H}_{2} \mathrm{O}$.

Les conditions du programme de la réaction PCR sont: une dénaturation initiale $94^{\circ} \mathrm{C}$ pendant 1 minutes, puis 35 cycles réactionnels (Dénaturation: $94^{\circ} \mathrm{C}$ pendant 40 secondes, Hybridation: $60^{\circ} \mathrm{C}$ pendant 30 secondes, Elongation: $72^{\circ} \mathrm{C}$ pendant 45 secondes) et une élongation finale $72^{\circ} \mathrm{C}$ pendant 10 minutes. La taille du fragment amplifié est de $476 \mathrm{pb}$. 


\section{5 Digestion enzymatique}

Le produit PCR obtenu est purifié puis digéré par l'enzyme de restriction BstzI en présence du tampon réactionnel à $37^{\circ} \mathrm{C}$ durant 2 heures. Le résultat de la digestion est soumis à une migration sur gel (3\% agarose) d'électrophorèse durant $1 \mathrm{~h} 30$.

\section{6 Analyses statistiques et bioinformatiques}

L'étude phylogénétique a été effectuée en utilisant le programme NTSYS-pc version 2.1 et la méthode UPGMA (Unweighted Pair Group Method using an Arithmetic average).

L'étude d'association génotype-phénotype a été effectuée moyennant le logiciel SPSS 17. Cette étude est utilisée pour évaluer les relations entre paires de variables notées selon une échelle binaire en utilisant le test Chi-deux $\left(\chi^{2}\right)$ à un degré de liberté. Les associations ayant une valeur $P<0.05$ sont considérées significatives. Les résultats de ce test sont vérifiés par le Test Exact de Fisher. Un test de Student a été également utilisé pour évaluer des associations significatives avec les variables quantitatives.

Une analyse multivariée a été également réalisée en utilisant la régression logistique binaire pour évaluer les relations entre une variable d'intérêt notée selon une échelle binaire et plusieurs autres variables.

\section{CONCLUSION}

L'étude du marqueur SNP localisé au niveau du gène calcium binding protein permet de valider la relation génotype-phénotype entre ce marqueur et quelques paramètres quantitatifs et qualitatifs, pouvant ainsi d'aboutir à l'identification d'éventuelles associations entre variabilité moléculaire et paramètres de qualité des huiles d'une part et de l'adaptation de l'arbre d'autre part. En effectuant des analyses bivariées quelques associations très significatives ont été observées pour le CALC (CG) avec le stade de maturation des fruits ( $p=$ $0,002)$ et la teneur en huile par fruit $(p=0,006)$. Trois importantes associations positives entre les variétés hétérozygotes CG-CALC et le contenu de l'huile en carotènes, le taux de cholestérol et l'acide linoléique ont été présentées, ce qui suggère que les variétés qui possèdent le génotype CG (principalement Chemlali) sont meilleurs que celles qui ont des génotypes homozygotes GG pour la capacité de produire ces composites. 


\section{ACKNOWLEDGEMENTS}

Ce travail a été financé par le Ministère de l'Enseignement Supérieur et de la Recherche Scientifique (Tunisie).

\section{REFERENCES}

[1] Ben Ayed R., Ennouri K., Ben Hassen H., Triki M.A., Rebai A. Comparaison between DNA-based, pomological and chemical markers accomplished by bioinformatic tools to distinguish within Tunisian olive cultivars. J Fundam Appl Sci., 2015, 7(3), 408-421.

[2] Hanana M., Hamrouni L., Cagnac O., Blumwald E. Mécanismes et stratégies cellulaires de tolérance à la salinité $(\mathrm{NaCl})$ chez les plantes. Environmental Review, 2011, 19, 121-140.

[3] Ranty B., Aldon D., Cotelle V., Galaud J.P., Thuleau P., Mazars C. Calcium Sensors as Key Hubs in Plant Responses to Biotic and Abiotic Stresses. Front Plant Sci., 2016, 16, 7-327. [4] Boudsocq M. Les senseurs de calcium dans la signalisation osmotique chez les plantes Plant calcium sensors in osmotic signalling. Biologie Aujourd'hui, 2010, 204 (1), 21-31.

[5] Shen C., Yang Y., Du L., Wang H. Calmodulin-binding transcription activators and perspectives for applications in biotechnology. Appl Microbiol Biotechnol, 2015,

[6] Hirschi K.D. Expression of Arabidopsis CAX1 in tobacco: altered calcium homeostasis and increase stress sensitivity Plant Cell., 1999, 11, 2113-2122.

[7] Virdi A.S., Singh S., Singh P. Abiotic stress responses in plants: roles of calmodulin-regulated proteins. Front Plant Sci., 2015, 14, 6:809.

[8] Reale S., Doveri S., Díaz A., Angiolillo A., Lucentini L., Pilla F., Martín A., Donini P., Lee D. SNP-based markers for discriminating olive (Olea europaea L.) cultivars. Genome, 2006, $15,1193-1209$.

[9] Knight M.R., Campbell A.K., Smith S.M., Trewavas A.J. Transgenic plant aequorin reports the effects of touch and cold-shock and elicitors on cytoplasmic calcium. Nature, 1991, $352,524-526$.

[10] Knight H., Trewavas A.J., Knight M.R. Calcium signalling in Arabidopsis thaliana responding to drought and salinity. Plant J., 1997, 12, 1067-78.

[11] Fabbri A., Hormaza J.I., Polito V.S. Random Amplified Polymorphic DNA Analysis of Olive (Olea europaea L.) Cultivars. J. Amer. Soc. Hort. Sci., 1995, 120(3): 538-542. 
[12] Ben Ayed R., Grati N., Moreau F. and Rebai A. Comparative study of microsatellite profiles of DNA from oil and leaves of two Tunisian olive cultivars European Food Research and Technology 2009, 229 (5), 757-762.

\section{How to cite this article:}

Ben Ayed R, Ennouri K and Rebai A . Involvement Of Snp Marker Located On The Calcium Binding Protein Gene In Adaptive Traits And Organoleptic Performances Of The Olive Tree. J. Fundam. Appl. Sci., 2018, 10(1), 328-343. 